\title{
Dectin-2-dependent host defense in mice infected with serotype 3 Streptococcus pneumoniae
}

Yukiko Akahori 1,5, Tomomitsu Miyasaka ${ }^{1,6}$, Masahiko Toyama ${ }^{1,7}$, Ikumi Matsumoto ${ }^{1}$, Anna Miyahara', Tong Zong ${ }^{1}$, Keiko Ishii ${ }^{1}$, Yuki Kinjo ${ }^{2}$, Yoshitsugu Miyazaki ${ }^{2}$, Shinobu Saijo ${ }^{3}$, Yoichiro Iwakura ${ }^{4}$ and Kazuyoshi Kawakami ${ }^{*}$

\begin{abstract}
Background: Streptococcus pneumoniae, a major causative bacterial pathogen of community-acquired pneumonia, possesses a thick polysaccharide capsule. Host defense against this bacterium is mediated by activation of innate immune cells that sense bacterial components. Recently, C-type lectin receptors (CLRs) have garnered much attention in elucidating the recognition mechanism of pathogen-derived polysaccharides.

Methods: In the present study, we first compared the clinical course and neutrophil accumulation in the lungs of Dectin-2 knock-out (KO) and wild type (WT) mice. Mice were infected intratracheally with a serotype 3 strain of $S$. pneumoniae, and S. pneumoniae bacterial engulfment by neutrophils and inflammatory cytokine and anti-pneumococcal polysaccharide-specific lgG levels were evaluated in bronchoalveolar lavage fluid (BALF). We also examined the effect of Dectin-2 deficiency on interleukin (IL)-12 production by bone marrow-derived dendritic cells (BM-DCs) stimulated with the bacterial components.

Results: S. pneumonia-infected Dectin-2KO mice had a shorter survival time, larger bacterial burden and lower interferon gamma (IFN- $\gamma$ ) production in the lungs than WT mice. Although neutrophilic infiltration in the lungs was equivalent between Dectin-2KO mice and WT mice, S. pneumonia engulfment by neutrophils was attenuated in Dectin-2KO mice compared to WT mice. The anti-pneumococcal polysaccharide-specific lgG and lgG3 levels in BALF were lower in Dectin-2KO mice than in WT mice. When BM-DCs were stimulated with S. pneumoniae culture supernatant or its Concanavalin A (ConA)-bound fraction, IL-12 production was abrogated in Dectin-2KO mice compared to WT mice.

Conclusions: We demonstrated that Dectin-2 is intimately involved in the host defense against infection with a serotype 3 strain of S. pneumoniae. Dectin-2-dependent IL-12 production may contribute to IFN- $\gamma$ synthesis and subsequent production of serotype-specific anti-capsular polysaccharide lgG after S. pneumoniae infection, which may promote S. pneumoniae bacterial opsonization for engulfment.
\end{abstract}

Keywords: Streptococcus pneumonia, Dectin-2, Neutrophils, Anti-capsular polysaccharide lgG, IFN- $\gamma$

\section{Background}

Streptococcus pneumoniae is recognized as a major bacterial agent that causes community-acquired pneumonia and other invasive diseases, such as bacteremia and meningitis [1,2]. Upon infection with S. pneumoniae, the innate mechanism of the early phase of immunity plays an important role in host defense, which is largely

\footnotetext{
*Correspondence: kawakami@med.tohoku.ac.jp

${ }^{1}$ Department of Medical Microbiology, Mycology and Immunology, Tohoku

University Graduate School of Medicine, Miyagi, Japan

Full list of author information is available at the end of the article
}

mediated by neutrophil-dependent immune responses. The infiltration of many neutrophils into the alveolar spaces eradicates the infection via an immunoglobulin $G$ (IgG)-mediated opsonophagocytic killing (OPK) mechanism and the production of reactive oxygen species (ROS) [3]. IgG3 that is specific for pneumococcal capsular polysaccharide, a thymus-independent type 2 (TI-2) antigen, is highly protective against infection with S. pneumoniae [4]. Recently, we reported similar findings that pneumococcal polysaccharide vaccine (PPV) immunization increases serotype 3-specific IgG3 serum levels, which facilitates 
survival after pneumococcal infection [5]. CXC chemokines, including macrophage inflammatory protein (MIP)2 and keratinocyte-derived chemokine (KC), a homologue of human interleukin (IL)-8, were involved in neutrophil accumulation at the inflammatory sites. Previous studies showed that death in mice challenged with S. pneumoniae was preceded by bacterial growth within 2 days after infection and was associated with a delayed increase in pulmonary MIP-2 levels and neutrophil recruitment [6].

DC-associated C-type lectin-2 (Dectin-2), a C-type lectin receptor, possesses a carbohydrate recognition domain for the $\mathrm{Ca}^{2+}$-dependent recognition of mannose oligosaccharides $[7,8]$. Dectin-2 invokes innate immune responses and subsequent adaptive immunity in fungal infection. Dectin-2-mediated recognition of Candida albicans leads to NF- $k B$ activation, which induces IL-1 $\beta$, IL-12 and IL-23 production by macrophages [7]. IL-1 $\beta$ and IL-23 potentiate neutrophil recruitment into the infected tissues through inducing IL-17A production [9-12]. T helper (Th)-1 cell-related cytokines, IL-12 and interferon gamma (IFN- $\gamma$ ), play a critical role in the neutrophil-mediated host defense against $S$. pneumoniae infection, which is correlated with MIP-2 and TNF- $\alpha$ production $[13,14]$. Mice with targeted disruption of the IL-12 or IFN- $\gamma$ gene are highly susceptible to pneumococcal pneumonia $[13,15]$.

In the present study, we aimed to determine the role of Dectin-2 in the neutrophil-mediated host defense to $S$. pneumoniae infection using mice with a genetic disruption of Dectin-2. We found that Dectin-2 knock out (KO) mice were more susceptible to this infection than wild type (WT) mice, and our results suggest that Dectin-2dependent IL-12 production may contribute to IFN- $\gamma$ synthesis and subsequent production of serotype-specific anti-capsular polysaccharide $\operatorname{IgG}$ after $S$. pneumoniae infection, which may promote opsonization of this bacterium for engulfment.

\section{Results}

Role of Dectin-2 in the host defense to pneumococcal infection

To clarify whether Dectin-2 deficiency affects earlyphase host protection against pneumococcal infection, we initially examined the susceptibility of Dectin-2KO mice to S. pneumoniae infection and compared it with WT mice by recording the survival rate of the infected mice and also the bacterial load in their lungs. Dectin$2 \mathrm{KO}$ mice had a lower survival rate $(17 \%$ by day 4 after intratracheal S. pneumoniae infection), whereas $67 \%$ of WT mice survived throughout the observation period (Fig. 1a). The difference in the survival rate was statistically significant. In addition, the number of live bacterial colonies was significantly lower in the lungs of WT mice than in Dectin-2KO mice on day 3 post-infection (Fig. 1b). These data indicate that Dectin-2 plays a critical role in early-phase host defense against pneumococcal infection.

\section{Role of Dectin-2 in the neutrophil-mediated host defense to pneumococcal infection}

Neutrophils rapidly accumulate at the most infected sites and they play a central role in eradicating bacteria after pulmonary S. pneumoniae infection [16]. Therefore, to address the role of Dectin-2 in neutrophil-mediated host defense against this bacterial pathogen, we first evaluated neutrophil recruitment in the infected lungs. A histological analysis showed no apparent difference in inflammatory cell infiltration in the lungs between WT and Dectin-2KO mice $12 \mathrm{~h}$ after infection with S. pneumoniae. When observed at a higher magnification,
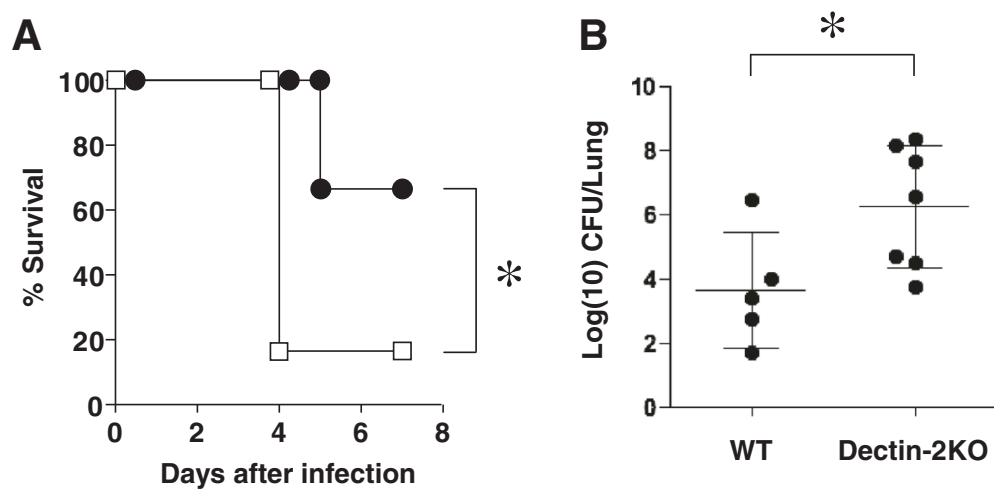

Fig. 1 Effect of Dectin-2 deficiency on S. pneumoniae infection. WT mice and Dectin-2KO mice were infected with S. pneumoniae. a The number of live mice was recorded daily. Closed circles, WT mice $(n=6)$; open squares, Dectin-2KO mice $(n=6)$. b Bacterial load in the lungs was examined on day 3 post-infection. Each symbol shows each mouse, and the bars indicate the mean \pm SD. Similar results were obtained in three independent experiments. ${ }^{*} p<0.05$ 
neutrophil accumulation in the alveolar spaces was shown to be comparable between these mice (Fig. 2a). In addition, the number of neutrophils in bronchoalveolar lavage fluid (BALF) was almost equivalent between WT and Dectin$2 \mathrm{KO}$ mice $12 \mathrm{~h}$ and $24 \mathrm{~h}$ after infection (Fig. $2 \mathrm{~b}$ ).

To further clarify the role of Dectin-2 in the neutrophil-mediated host defense, we evaluated the phagocytosis rate and phagocytosis index of these cells in BALF $12 \mathrm{~h}$ and $24 \mathrm{~h}$ after infection. As shown in Fig. $3 \mathrm{a}$, the rate of neutrophils engulfing pneumococcus was significantly lower in Dectin-2KO mice than in WT mice at both time points, although the average number of pneumococcus per engulfed neutrophil did not differ largely between these mice (Fig. 3b).

\section{Role of Dectin-2 in the production of proinflammatory} cytokines and chemokines after pneumococcal infection To clarify the role of Dectin-2 in the host response to $S$. pneumoniae infection, we compared the production of proinflammatory cytokines and chemokines, such as IL$1 \beta$, TNF- $\alpha$, IL-6, IFN- $\gamma$, IL-17A, and MIP-2, in BALF between WT and Dectin-2KO mice $12 \mathrm{~h}$ after infection. As shown in Fig. 4, the production of IFN- $\gamma$ was significantly attenuated in Dectin-2KO mice compared with WT mice, although there was no significant difference in the production of other cytokines and chemokines.

To define the cellular source of IFN- $\gamma$ production, we used flow cytometry to examine the intracellular expression of this cytokine in various cells in the lungs of WT mice $12 \mathrm{~h}$ after infection. IFN- $\gamma$ was expressed only in exudate macrophages, but not in alveolar macrophages, neutrophils, natural killer (NK) cells, NKT cells, $\gamma \delta \mathrm{T}$ cells, CD4 ${ }^{+} \mathrm{T}$ cells or $\mathrm{CD}^{+} \mathrm{T}$ cells (Fig. 5).

\section{Reduced production of PPS3-specific Ab in Dectin-2KO mice}

Serotype-specific IgG against capsular polysaccharides plays a critical role as an opsonin in the phagocytosis of S. pneumoniae by neutrophils, which strongly promotes its eradication [17]. IgG3 is a major subclass of IgG produced under the stimulation of TI-2 Ags such as pneumococcal capsular polysaccharides [18]. We next measured PPS3-specific IgG and IgG3 levels in BALF $24 \mathrm{~h}$ after infection. As shown in Fig. $6 \mathrm{a}$ and b, the levels of PPS3-specific IgG and IgG3 in BALF were significantly increased $24 \mathrm{~h}$ after infection with $S$. pneumoniae in both WT and Dectin-2KO mice, and their levels were significantly lower in Dectin-2KO mice compared with WT mice. By contrast, PPS3-specific IgM levels in BALF were low, and there was no difference in the levels between WT and Dectin-2KO mice (Fig. 6c).

\section{Role of Dectin-2 in the activation of dendritic cells upon stimulation with $S$. pneumoniae}

To address the role of Dectin-2 in the cellular response to $S$. pneumoniae, we examined how the lack of Dectin2 affected the production of IL-12p40 by bone marrowderived dendritic cells (BM-DCs) upon stimulation with viable S. pneumoniae, lysates or culture supernatant from this bacterium. As shown in Fig. 7a, IL-12p40 production by BM-DCs was almost comparable between WT and Dectin-2 KO mice, when stimulated with viable

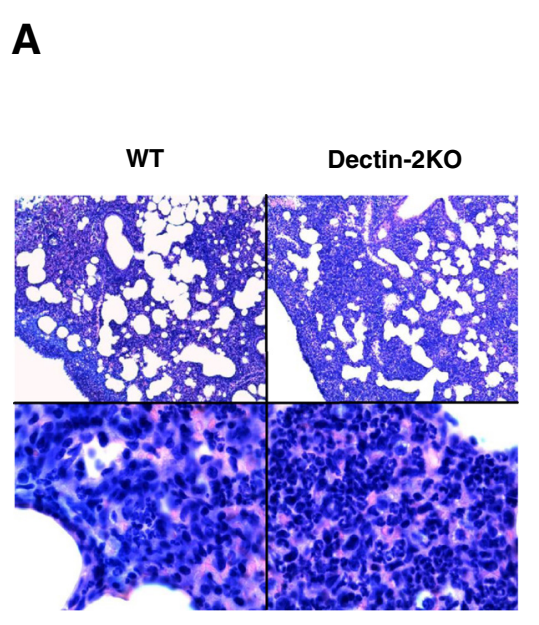

B
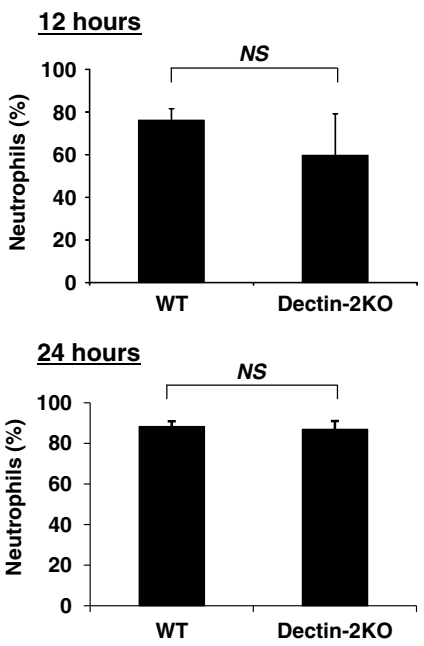

Fig. 2 Equivalent level of neutrophil infiltration in lungs between WT and Dectin-2KO mice. WT mice and Dectin-2KO mice were infected with S. pneumoniae. a The lung section obtained $12 \mathrm{~h}$ post-infection was stained with hematoxylin-eosin (H-E), and then observed using light microscopy. Original magnifications: $\times 100$ and $\times 1000$. b Cells in BALF at $12 \mathrm{~h}$ and $24 \mathrm{~h}$ post-infection were stained with Diff-Quick, and the composition of neutrophils was then quantified. Each group consists of five to seven mice. Similar results were obtained in three independent experiments. NS, not significantly different 

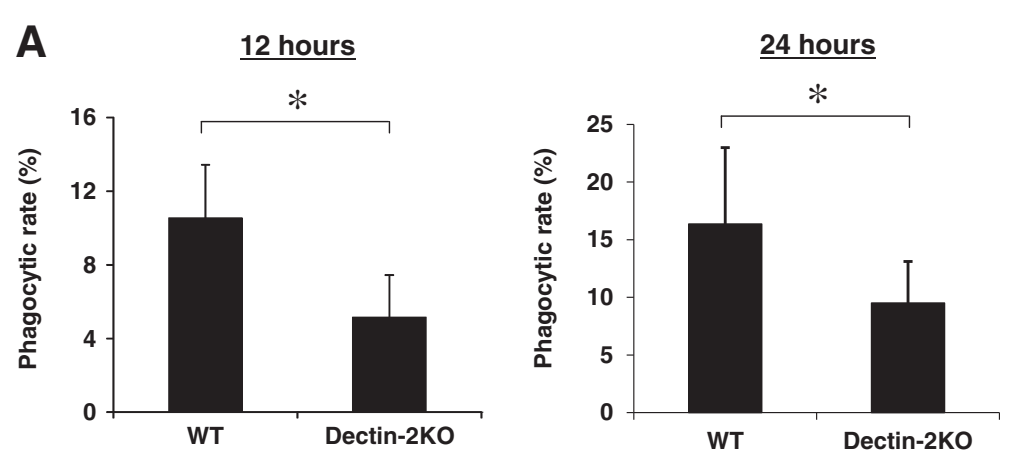

B
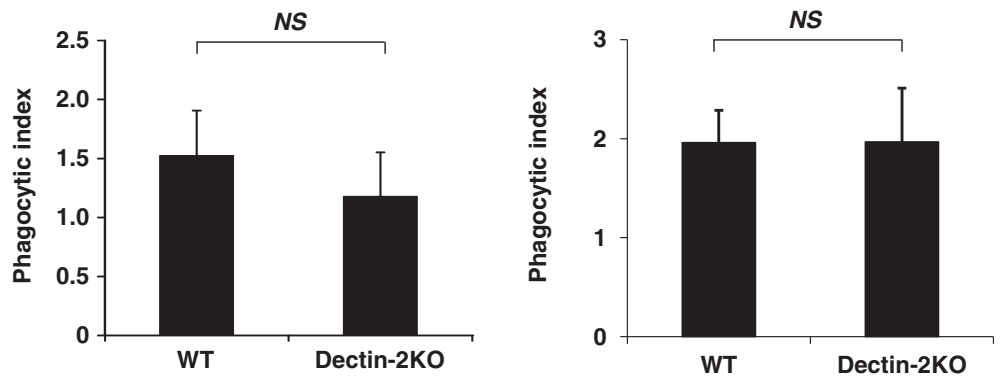

Fig. 3 Effect of Dectin-2 deficiency on the engulfment of pneumococcus by neutrophils. WT mice and Dectin-2KO mice were infected with S. pneumoniae. The phagocytic rate (a) and phagocytic index (b) of neutrophils in BALF were calculated $12 \mathrm{~h}$ and $24 \mathrm{~h}$ after infection. The number of neutrophils was estimated by multiplying the total cell count by its proportion identified in morphological analysis. Each group consists of five to seven mice. Similar results were obtained in three independent experiments. ${ }^{*}, p<0.05$; NS, not significantly different
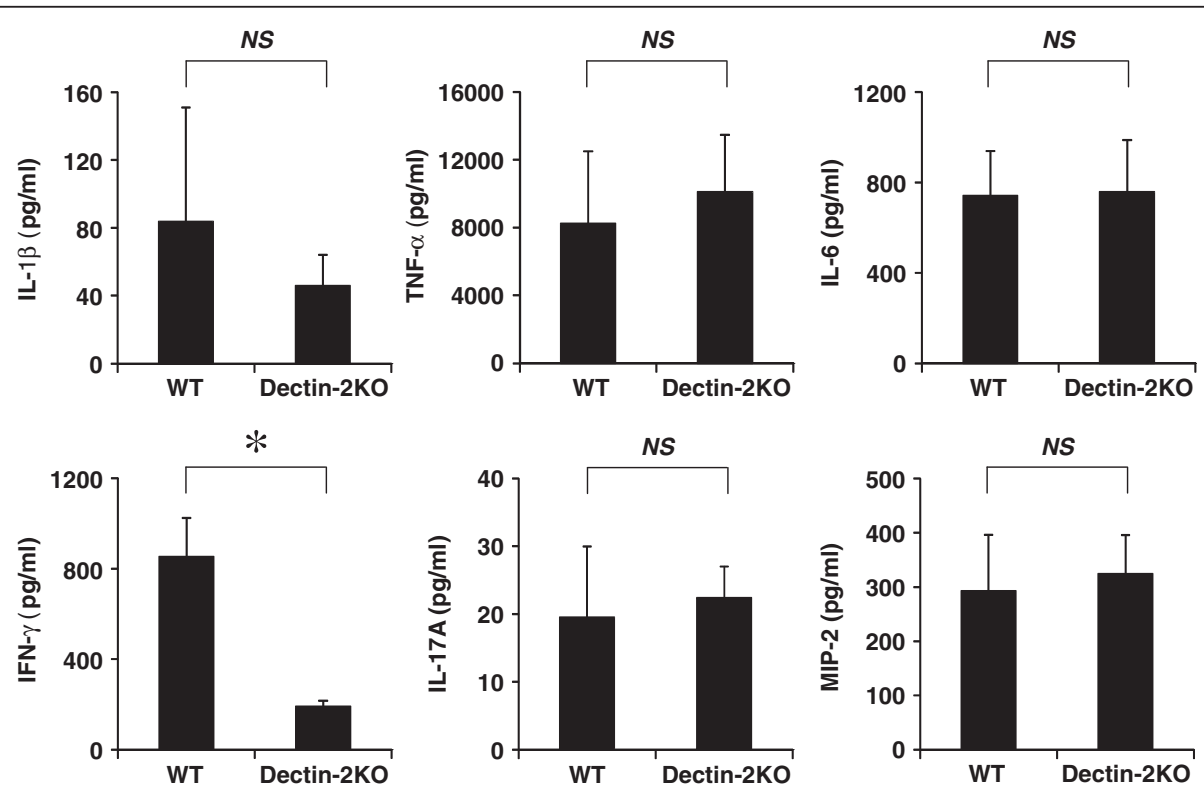

Fig. 4 Effect of Dectin-2 deficiency on cytokine production in the lungs after pneumococcal infection. WT mice and Dectin-2KO mice were infected with S. pneumoniae. Cytokine concentrations in BALF were measured $12 \mathrm{~h}$ post-infection. Each group consists of five to seven mice. Similar results were obtained in three independent experiments. ${ }^{*}, p<0.05$; NS, not significantly different 

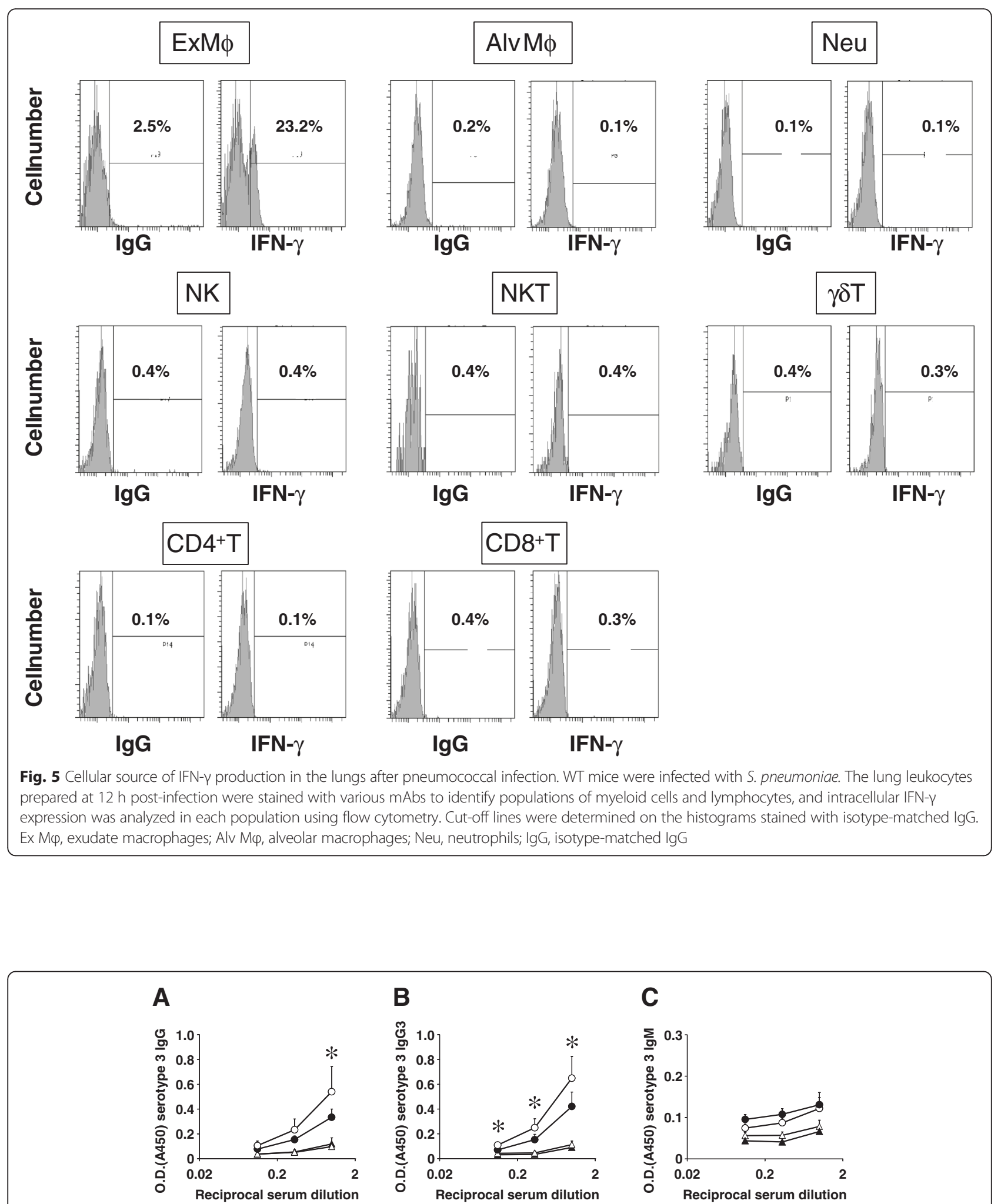

Fig. 6 Reduced production of serotype-specific Ab against pneumococcal capsular polysaccharides in Dectin2KO mice. Anti-PPS3 lgG (a), lgG3 (b) and $\operatorname{lgM}(\mathbf{c})$ concentrations in BALF before infection or $24 \mathrm{~h}$ post-infection were measured as OD450 values at $\times 1, \times 3$ and $\times 9$ dilution, respectively. Each group consists of six to seven mice. Similar results were obtained in three independent experiments. *, $p<0.05$, compared with Dectin-2KO mice $24 \mathrm{~h}$ post-infection. Open circles, WT mice $24 \mathrm{~h}$ post-infection; closed circles, Dectin-2KO mice $24 \mathrm{~h}$ after infection; open triangles, WT mice before infection; closed triangles, Dectin-2KO mice before infection 

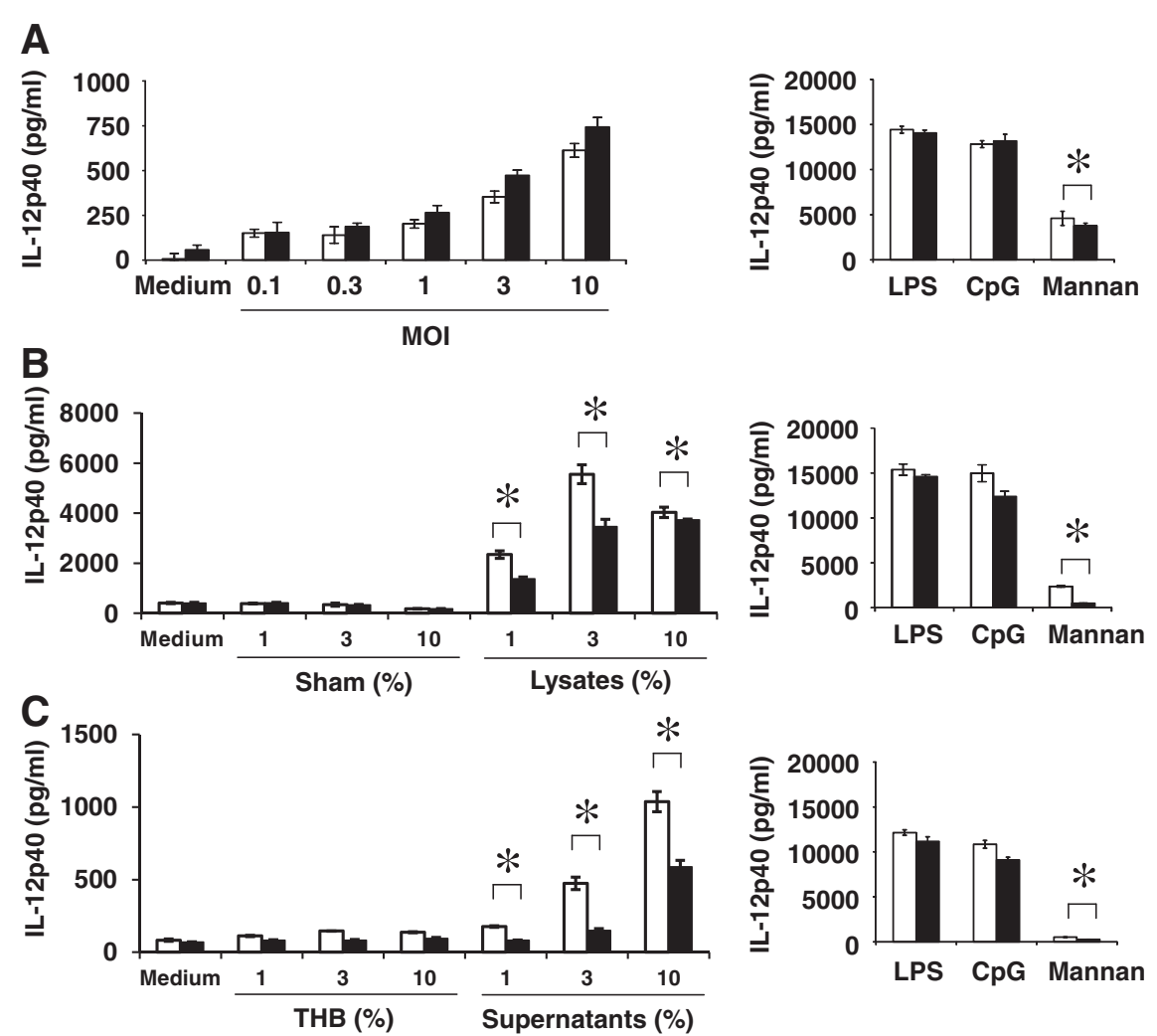

Fig. 7 Effect of Dectin-2 deficiency on IL-12p40 production by BD-DCs upon stimulation with S. pneumoniae. BM-DCs from WT mice (open columns) or Dectin-2KO mice (solid columns) were cultured with live S. pneumoniae (a); lysates from S. pneumoniae or sham-operated PBS (Sham)

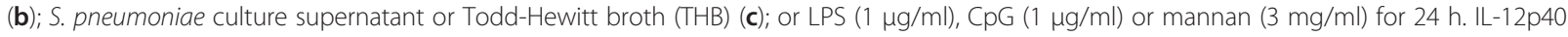
concentrations in the culture supernatants were measured using ELISA. Each column shows the mean \pm SD of triplicate culture. Similar results were obtained in three independent experiments. MOI, multiplicity of infection. ${ }^{*}, p<0.05$; NS, not significantly different

pneumococcus, whereas IL-12p40 was not produced by BM-DCs stimulated with heat-killed bacteria (data not shown). By contrast, IL-12p40 synthesis by BM-DCs was significantly reduced in Dectin-2KO mice compared with WT mice, when stimulated with $S$. pneumoniae lysates at lower doses (Fig. 7b). Similar results were obtained when BM-DCs were stimulated with S. pneumoniae culture supernatant (Fig. 7c).

To determine which S. pneumoniae molecule is recognized by Dectin-2, we examined how depletion of the Concanavalin A (ConA)-bound fraction in culture supernatants affects IL-12p40 synthesis by BM-DCs. IL-12p40 synthesis by BM-DCs from WT mice was abolished when stimulated with the culture supernatant that was depleted of the ConA-bound fraction (Fig. 8a). In further experiments, we examined whether the ConA-bound fraction in culture supernatant stimulated BM-DCs and whether this activity was dependent on Dectin-2. As shown in Fig. 8b, the ConA-bound fraction induced IL-12p40 production by BM-DCs from WT mice and this activity was completely abrogated in BM-DCs from Dectin-2KO mice, similar to the response caused by mannan.

\section{Discussion}

In the present study, we evaluated the role of Dectin-2 in the neutrophil-mediated host defense to pneumococcal infection. Our data indicate that a defect in Dectin-2 rendered mice highly susceptible to a serotype 3 strain of S. pneumoniae, as shown by elevated mortality and an increased bacterial burden in the lungs. It is well documented that neutrophil-predominant inflammatory responses play a pivotal role in eradicating this bacterium [3]. Therefore, we predicted that a defect of Dectin-2 expression impaired the recruitment of neutrophils and clearance of S. pneumoniae in the lungs after infection. Dessing and co-workers previously demonstrated that TLR2- and TLR4-mediated recognition of pneumococcal components induced the production of proinflammatory cytokines, such as IL- 6 and IL-1 $\beta$, and chemokines, such as MIP-2 and KC, which are critical for neutrophil accumulation in inflamed tissues $[19,20]$. They also found that a lack of TLR2 led to earlier death from pneumococcal meningitis [21]. In the present study, however, neutrophil accumulation and TNF- $\alpha$, IL-1 $\beta$, IL-17A and MIP-2 synthesis in the lungs after infection with $S$. pneumoniae were not significantly different between WT and Dectin-2KO 


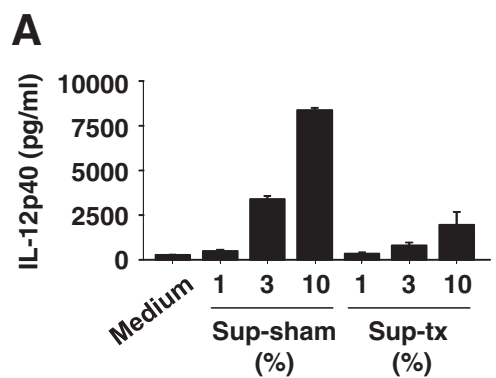

B

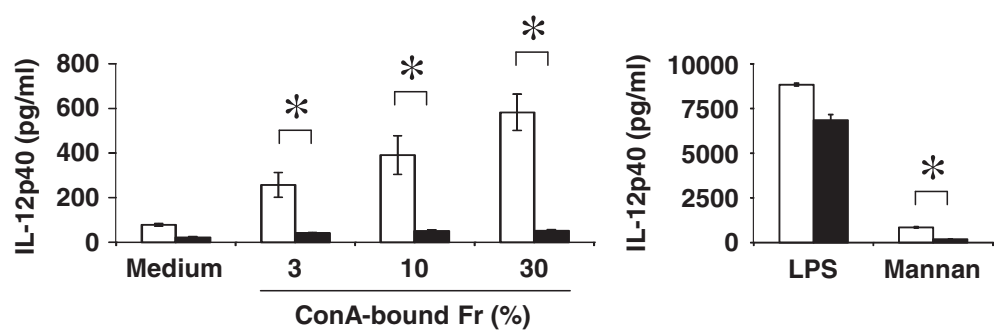

Fig. 8 Dectin-2-dependent IL-12p40 production by BM-DCs in the ConA-bound fraction of S. pneumoniae culture supernatant. a BM-DCs derived from WT mice were stimulated with the ConA-Sepharose4B-treated or sham-treated supernatant from S. pneumoniae or mannan for $24 \mathrm{~h}$. $\mathbf{b}$ BM-DCs from WT mice (open columns) or Dectin-2KO mice (solid columns) were cultured with the ConA-Sepharose4B-bound fraction of S. pneumoniae culture supernatants, LPS $(1 \mu \mathrm{g} / \mathrm{ml})$ or mannan $(3 \mathrm{mg} / \mathrm{ml})$ for $24 \mathrm{~h}$. The IL-12p40 concentration in the culture supernatants was measured by ELISA. Each column shows the mean \pm SD of triplicate culture. Similar results were obtained in three independent experiments. Sup-sham, sham-treated supernatants; Sup-tx, ConA-Sepharose4B-treated supernatants; Mannan-sham, sham-treated mannan; Mannan-tx, ConA-Sepharose4B-treated mannan; ConA-bound Fr, CpnA-Sepharose4B-bound fraction. ${ }^{*} p<0.05$

mice. By contrast, the engulfment of pneumococcus by neutrophils was significantly impaired in Dectin-2KO mice compared with WT mice. These results suggest that Dectin-2 may be involved in the phagocytic killing of $S$. pneumoniae by neutrophils rather than in the accumulation of these cells. Recently, a similar observation was reported by Albiger et al., which addressed the role of TLR9 in the host defense against S. pneumoniae infection [22]. TLR9KO mice were significantly more susceptible to this infection than WT mice, probably as a result of the impaired phagocytic killing of this bacterium by macrophages.

Earlier observations demonstrated that the prognosis of bacteremia following pneumococcal pneumonia was improved in the early phase of infection, which might be related to the development of specific anti-capsular $\mathrm{Ab}$ [23]. Opsonization of this bacterium by IgG specific to the capsular polysaccharides is a key step in the neutrophil-mediated host defense against this infection $[17,24,25]$. TI-2 Ag, including pneumococcal capsular polysaccharides, typically elicits a rapid extrafollicular IgG response with limited isotype class switching from IgM to IgG, affinity maturation of immunoglobulin and memory B cell response [26]. However, our recent study demonstrated that the serum levels of serotype-specific IgG against pneumococcal polysaccharide Ag were increased in mice immunized with PPV [5]. Similar observations were reported by other investigators $[27,28]$. Pneumococcal capsular polysaccharides have been shown to produce serotype-specific IgG, irrespective of their features as TI-2 Ag. In a clinical study, Verbinnen and co-workers demonstrated that B-1-like B cells producing serotype-specific IgG were significantly increased in the peripheral blood of healthy volunteers as early as day 5 after immunization with PPV [29]. In addition, Haas and co-workers demonstrated that B-1 B cells were involved in the protection during early responses against $S$. pneumoniae infection through the synthesis of IgG3 to TI-2 Ag [30]. Although B cell subsets producing IgG3 remain to be examined, the present data demonstrated that Dectin-2 was involved in the production of serotype-specific IgG3 in BALF as early as $24 \mathrm{~h}$ after infection was significantly reduced in Dectin-2KO mice compared with WT mice.

Earlier investigations reported the involvement of IFN$\gamma$ in isotype class switching of IgM to IgG3 in B cells upon stimulation with TI-2 Ag [31], although in the current study, it remains unclear whether IgG3 was produced as a result of class-switching from IgM because of equivalent anti-PPS3 IgM in BALF between WT and Dectin-2KO mice. In addition, Marchi and co-workers demonstrated that IFN- $\gamma$ enhanced opsonized zymosan phagocytosis and ROS release by neutrophils [32]. Recently, we reported that a defect in Dectin-2 led to reduced IFN- $\gamma$ production by NKT cells during immunization with PPV 
[5]. Similarly, in the present study, IFN- $\gamma$ synthesis in the lungs was significantly attenuated in Dectin-2KO mice compared with WT mice after infection with S. pneumoniae. Thus, IFN- $\gamma$ may play a pivotal role in regulating the phagocytic killing by neutrophils as a downstream event in Dectin-2-mediated recognition of pneumococcal capsular polysaccharides. Previously, we demonstrated that IL-12 plays an important role in host defense against pneumococcal infection by promoting the production of IFN- $\gamma$ [13]. In the present study, Dectin-2 was essential for triggering IL-12p40 production by DCs upon simulation with S. pneumoniae culture supernatant, which supports the above hypothesis.

The ConA-bound fraction in S. pneumoniae culture supernatant induced IL-12p40 production by BM-DCs, and this activity was completely abrogated when BM-DCs were derived from Dectin-2KO mice. These results suggest that certain ConA-bound moieties of the capsular polysaccharides may be involved in Dectin-2-mediated recognition of S. pneumoniae. In earlier studies, Lee and co-workers demonstrated that Dectin-2 had an ability to bind Glc-, Gal-, GlcNAc- and GalNAc-BSA, in addition to the usual Man- and Fuc-BSA [33]. Additionally, ConA has been known to bind molecules that contain $\alpha$-Dmannose, $\alpha$-D-glucose and sterically related residues with available C-3, C-4 or C-5 hydroxyl groups [34]. These previous findings suggest that some glucosyl residue in the capsular polysaccharides might be recognized by Dectin- 2 . Further investigations are necessary to define the precise polysaccharide structure that contributes to S. pneumoniae recognition through Dectin-2.

Among different S. pneumoniae serotypes, capsular polysaccharide structures are not identical [35]. McGreal and co-workers previously reported that serotype 3 capsular polysaccharide inhibited the interaction between Dectin-2 and mannans, whereas other serotypes, such as 2, $9 \mathrm{~V}, 14,18 \mathrm{C}$ and $19 \mathrm{~F}$, did not show such an effect [36], suggesting a distinct role for Dectin-2 in the recognition of different $S$. pneumoniae serotypes. In the present study, we used only a serotype 3 strain of $S$. pneumoniae, and therefore, these findings may not be generalizable to all pneumococcal infections.

\section{Conclusions}

In the present study, we demonstrate that Dectin- $2 \mathrm{KO}$ mice were more susceptible to infection with a serotype 3 strain of S. pneumoniae than WT mice, as shown by a shorter survival time, larger bacterial burden and lower IFN- $\gamma$ production in the lungs of Dectin-2KO mice. Our results suggest that Dectin-2-dependent IL-12 production may contribute to IFN- $\gamma$ synthesis and subsequent production of serotype-specific anti-capsular polysaccharide IgG after S. pneumoniae serotype 3 infection, which may promote opsonization of this bacterium for engulfment. Thus, the present study may provide important implications for better understanding in the host defense mechanism against $S$. pneumoniae serotype 3 and for developing more effective vaccine strategies against this infection.

\section{Methods \\ Mice}

Dectin-2KO mice were generated by homologous recombination of the Clec $4 n$ gene as described previously [7]. WT littermate mice of the Dectin-2KO mice were used as controls. Male or female mice at 6 to 8 weeks of age were used for the experiments. The mice were bred under specific pathogen-free conditions at the Animal Facility, Tohoku University Graduate School of Medicine (Sendai, Japan). All experimental procedures involving animals followed the Regulations for Animal Experiments and Related Activities at Tohoku University and were approved by the ethics committees of Tohoku University.

\section{Bacteria}

A serotype-3 clinical strain of $S$. pneumoniae, designated as URF918, was established from a patient with pneumococcal pneumonia [37]. The bacteria were cultured in Todd-Hewitt broth (Difco, Detroit, MI, USA) at $37^{\circ} \mathrm{C}$ in a $5 \% \mathrm{CO}_{2}$ incubator, harvested at the mid-log phase of growth and then washed twice in phosphate buffered saline (PBS). The inoculum was stored at $-80{ }^{\circ} \mathrm{C}$ until use.

\section{S. pneumoniae infection}

WT or Dectin-2KO mice were anaesthetized by an intraperitoneal injection of $70 \mathrm{mg} / \mathrm{kg}$ pentobarbital (Abbott Laboratory, North Chicago, IL, USA) and restrained on a small board. Live S. pneumoniae $\left(0.75-3 \times 10^{5}\right.$ colony forming units (CFU)) at $50 \mu \mathrm{l}$ per mouse were inoculated by insertion of a $24 \mathrm{G}$ intravenous (IV) catheter (Terumo, Tokyo, Japan) into the trachea. Colony counts were performed to confirm the accuracy of inoculum CFU as a means to determine $\mathrm{CFU} / \mathrm{ml}$ for $S$. pneumoniae using a $5 \%$ sheep blood tryptic soy agar plate (Nissui Pharmaceutical Co., Ltd., Tokyo, Japan).

\section{Enumeration of viable S. pneumoniae}

WT and Dectin-2KO mice were sacrificed on day 3 postinfection, and the lungs were dissected carefully and excised. They were then homogenized in $5 \mathrm{ml}$ of PBS by teasing with stainless mesh at room temperature. The homogenates $(100 \mu \mathrm{l})$ were diluted in twofold series using sterile half saline and inoculated onto a $5 \%$ sheep blood tryptic soy agar plate (Nissui Pharmaceutical Co., Ltd.). The homogenate was then cultured for $24 \mathrm{~h}$ at $37^{\circ} \mathrm{C}$ in $5 \% \mathrm{CO}_{2}$, and the number of colonies was counted. 


\section{Lung histology}

Lungs were isolated from WT or Dectin-2KO mice $12 \mathrm{~h}$ after pneumococcal infection, and fixed in $10 \%$ buffer formalin, dehydrated and embedded in paraffin. Sections were cut and stained with hematoxylin-eosin (H-E) or Gram stain at the Biomedical Research Core, Animal Pathology Platform of Tohoku University Graduate School of Medicine (Sendai, Japan).

\section{Preparation of BALF}

BALF samples from WT or Dectin-2KO mice were collected as described below. Briefly, after bleeding under anesthesia with isoflurane, the chest was opened and the trachea was cannulated with the outer sheath of a $22 \mathrm{G}$ IV catheter/needle unit (Terumo), followed by lung lavage three times with $1 \mathrm{ml}$ of chilled PBS. Then, $1 \times 10^{5}$ cells were centrifuged onto a glass slide using StatSpin Cytofuge 2 (Iris Sample Processing, Franklin, MA, USA), and then stained with Diff-Quick or Gram stain. After centrifugation of BALF, supernatants were stored at $-80{ }^{\circ} \mathrm{C}$ for cytokine assay. To analyze the leukocyte fraction and $S$. pneumoniae phagocytosis by neutrophils, at least 500 cells were examined using light microscopy.

\section{Measurement of cytokine concentration}

TNF- $\alpha$ and IFN- $\gamma$ levels in the BAL fluids, and IL-12p40 levels in culture supernatants were determined by enzymelinked immunosorbent assay (ELISA) using capture and biotinylated developing antibodies (BD Biosciences, Franklin Lakes, NJ, USA). Collected BALF was assessed using ELISA kits for IL-1 $\beta$, IL-6, IL-17A (eBioscience, San Diego, CA, USA) and MIP-2 (RayBiotech, Norcross, GA, USA). The detection limits of assays for TNF- $\alpha$, IFN- $\gamma$, IL-12p40, IL$1 \beta$, IL-6, IL-17A and MIP-2 were $50,15,15,8,4,4$ and $2.74 \mathrm{pg} / \mathrm{ml}$, respectively.

\section{In vivo neutrophil phagocytosis}

Twelve hours after pneumococcal infection, WT and Dectin-2KO mice were sacrificed and BALF was collected. The cells were spun onto glass slides, and the phagocytic index and phagocytic rate were examined by light microscopy following Gram staining. The phagocytic rate was calculated as follows: (number of neutrophils engulfing pneumococcus/total number of neutrophils) $\times 100$. The phagocytic index was calculated as follows: (number of phagocytized pneumococcus/total number of neutrophils engulfing pneumococcus).

\section{Measurement of serotype-specific Antibodies}

BALF samples were collected before infection or $24 \mathrm{~h}$ post-pneumococcal infection. The quantities of serotypespecific antibodies $(\mathrm{Ab})$ against pneumococcal polysaccharide type 3 (PPS3) in BALF were measured by ELISA. Microtiter plates (Nunc A/S, Roskilde, Denmark) were coated with $3 \mu \mathrm{g} / \mathrm{ml}$ of PPS3 (American Type Culture Collection, Manassas, VA, USA) in PBS for $1 \mathrm{~h}$ at $37^{\circ} \mathrm{C}$. Before testing, serum samples were diluted with $0.05 \%$ skim milk PBS. HRP-conjugated goat anti-mouse IgG, IgG3 or IgM antibodies (Southern Biotechnology Associates, Birmingham, AL, USA) diluted with 1:4000 were used as detection Ab. The concentrations of IgG, IgG3 and IgM were determined based on the absorbance at $450 \mathrm{~nm}$.

\section{Analysis of intracellular IFN- $\gamma$ expression}

Lung leukocytes, prepared as previously described [38], were stained with APC-anti-CD3e mAb (clone 145-2C11, Biolegend, San Diego, CA, USA), Pacific Blue-anti-CD4 $\mathrm{mAb}$ (clone GK1.5, Biolegend), APC/Cy7-anti-CD8 mAb (clone 53-6.7, Biolegend), PE/Cy7-anti-NK1.1 mAb (clone PK136, Biolegend) and PE-anti-TCR $\delta \mathrm{mAb}$ (clone GL3, Biolegend). The cells were also stained with PE-antiCD11b mAb (clone M1/70, Biolegend), PE/Cy7-anti-Gr-1 mAb (clone RB6-8C5, Biolegend) and Pacific Blue-anti$\mathrm{F} 4 / 80 \mathrm{mAb}$ (clone BM8, Biolegend). After washing twice, the cells were incubated in the presence of cytofix/ cytoperm (BD Biosciences), washed twice in BD perm/ wash solution (BD Biosciences) and stained with FITCanti-IFN- $\gamma$ mAb (clone XMG1.2, Biolegend). Isotypematched IgG was used for control staining. The stained cells were analyzed using a BD FACS Canto ${ }^{\mathrm{TM}}$ II flow cytometer (BD Biosciences). Data were collected from 20,000 to 30,000 individual cells using forward-scatter and side-scatter parameters to set a gate on the lymphocyte or myeloid cell population.

\section{Preparation and culture of dendritic cells}

Bone marrow cells from WT mice and Dectin-2KO mice were cultured at $2 \times 10^{5} / \mathrm{ml}$ in $10 \mathrm{ml}$ RPMI1640 medium (Nippro, Osaka, Japan) supplemented with $10 \%$ fetal calf serum (FCS) (Biowest, Nuaillé, France), $100 \mathrm{U} / \mathrm{ml}$ penicillin $\mathrm{G}, 100 \mu \mathrm{g} / \mathrm{ml}$ streptomycin, $2 \mathrm{mM}$ L-glutamine and $50 \mu \mathrm{M}$ 2-mercaptoethanol (Sigma-Aldrich, St. Louis, MO, USA) containing $20 \mathrm{ng} / \mathrm{ml}$ murine granulocytemacrophage colony-stimulating factor (GM-CSF; Wako Pure Chemical Industries, Ltd., Osaka, Japan). On day 3, $10 \mathrm{ml}$ of the same medium was added, followed by replacement of half of the medium with GM-CSFcontaining culture medium on day 6 . On day 8 or 9 , non-adherent cells were collected and used as BM-DCs. BM-DCs were cultured at $1 \times 10^{5} / \mathrm{ml}$ with various stimuli at $37{ }^{\circ} \mathrm{C}$ in a $5 \% \mathrm{CO}_{2}$ incubator for $24 \mathrm{~h}$. Lipopolysaccharide (LPS) prepared from Escherichia coli O-111 (SigmaAldrich), phosphorothioated CpG1826 oligonucleotide (Hokkaido System Science, Sapporo, Japan), and mannan from Saccharomyces cerevisiae (Sigma-Aldrich) were used as controls. 


\section{Preparation of S. pneumoniae homogenates}

S. pneumoniae were grown on $5 \%$ sheep blood tryptic soy agar plate (Nissui Pharmaceutical Co., Ltd., Tokyo, Japan) at $37{ }^{\circ} \mathrm{C}$ in a $5 \% \mathrm{CO}_{2}$ incubator for $24 \mathrm{~h}$. Harvested colonies were crushed in PBS using $0.1 \mathrm{~mm}$ glass beads and a Multi-Beads Shocker (Yasuikikai, Osaka, Japan) at $2500 \mathrm{rpm}$ and $4{ }^{\circ} \mathrm{C}$ for 40 cycles (30 s on/30 s off), passed through a $40 \mu \mathrm{m}$ nylon mesh filter (Becton, Dickinson and Company, Franklin Lakes, NJ, USA), and then stored at $-80^{\circ} \mathrm{C}$ until use. Sham-operated PBS were treated identically without $S$. pneumoniae and used as controls.

\section{Preparation of S. pneumoniae culture supernatants}

S. pneumoniae was diluted with half saline until the turbidity reached 0.5 using the McFarland standard, inoculated in 19 volumes of Todd-Hewitt broth (Difco) and then incubated on an orbital shaker $(150 \mathrm{rpm})$ at $37{ }^{\circ} \mathrm{C}$ in a $5 \% \mathrm{CO}_{2}$ incubator for $24 \mathrm{~h}$. The culture supernatants were centrifuged, passed through a $0.45 \mu \mathrm{m}$ membrane filter (Sartorius, Göttingen, Germany) and stored at $-80{ }^{\circ} \mathrm{C}$ until use. Todd-Hewitt broth incubated without S. pneumoniae was used as a control.

\section{ConA-affinity chromatography of S. pneumoniae culture supernatants}

ConA-Sepharose4B (GE Healthcare Bio-Sciences AB, Uppsala, Sweden) was prepared according to the manufacturer's instructions. Briefly, a polystyrene column $(0.6 \times 18 \mathrm{~cm}$; bed volume, $5 \mathrm{ml})$ was packed with ConASepharose4B. The column was washed with $50 \mathrm{ml}$ of binding buffer (20 mM Tris- $\mathrm{HCl}, 0.5 \mathrm{M} \mathrm{NaCl}, \mathrm{pH}$ 7.4) for regeneration and re-equilibration. S. pneumoniae culture supernatants were applied to the column continuously at a flow rate of $3 \mathrm{ml} / \mathrm{h}$. The bound-fractions were eluted with an elution buffer of 0.4 M methyl- $\alpha-D-m a n-$ nopyranoside (Sigma-Aldrich) in $20 \mathrm{mM}$ Tris- $\mathrm{HCl}$ at pH 7.4 and with $0.5 \mathrm{M} \mathrm{NaCl}$, and dialyzed against PBS using a membrane with a 2-kDa molecular weight cutoff (Thermo Fisher Scientific Inc., IL, USA). All the chromatographic operations and dialysis were performed at $4{ }^{\circ} \mathrm{C}$. To collect the ConA-unbound fraction, S. pneumoniae culture supernatants were incubated with ConASepharose $4 \mathrm{~B}$ for $15 \mathrm{~min}$ at room temperature, and then the ConA-Sepharose4B beads were removed using centrifugation. Sham treatment was performed using Sepharose4B beads. Similarly, mannan received ConA-Sepharose4B or sham treatment.

\section{Statistical analysis}

Statistical analysis was conducted using GraphPad Prism 5 software (GraphPad Software, La Jolla,CA,USA). Data are presented as the mean \pm standard deviation (SD). Differences between the two groups were tested using a two-tailed analysis in an unpaired Student's $t$-test.
Survival data was analyzed using the Kaplan-Meier log rank test. A $p$ value less than 0.05 was considered significant.

\section{Abbreviations}

CLRs: C-type lectin receptors; KO: Knock-out; WT: Wild type;

BALF: Bronchoalveolar lavage fluid; BM-DCs: Bone marrow-derived dendritic cells; OPK: Opsonophagocytic killing; ROS: Reactive oxygen species; TI-

2: Thymus-independent type 2; PPV: Pneumococcal polysaccharide vaccine; MIP: Macrophage inflammatory protein; KC: Keratinocyte-derived

chemokine; PPRs: Pattern recognition receptors; PPS3: Pneumococcal polysaccharide type 3; FCS: Fetal calf serum; LPS: Lipopolysaccharide;

ConA: Concanavalin A.

\section{Competing interests}

The authors have no financial conflicts of interest.

\section{Authors' contributions}

YA, TM, MT, IM, AM, TZ and KI performed the experiments and analyzed the data; SS and YI contributed the Dectin-2KO mice; TM, YK, YM and KK conceived and designed the experiments; and TM and KK wrote the manuscript. All authors read and approved the final manuscript.

\section{Acknowledgements}

This work was supported in part by grants from the Ministry of Health, Labour and Welfare of Japan (H22-SHINKOU-IPPAN-014 to KK and H25SHINKOU-WAKATE-005 to YK), a grant from the Japan Agency for Medical Research and Development, AMED (the Research Program on Emerging and Re-emerging Infectious Diseases), aid funding from the Takeda Science Foundation to YK, and Joint Usage/Research Program of Medical Mycology Research Center, Chiba University [15-19] to KK.

\section{Author details}

${ }^{1}$ Department of Medical Microbiology, Mycology and Immunology, Tohoku University Graduate School of Medicine, Miyagi, Japan. ${ }^{2}$ Department of Chemotherapy and Mycoses, National Institute of Infectious Diseases, Tokyo, Japan. ${ }^{3}$ Division of Molecular Immunology, Medical Mycology Research Center, Chiba University, Chiba, Japan. ${ }^{4}$ Center for Animal Disease Models, Research Institute for Biomedical Sciences, Tokyo University of Science, Chiba, Japan. ${ }^{5}$ Present address: Japanese Red Cross Society, Tokyo, Japan. ${ }^{6}$ Present address: Department of Pathophysiology, Tohoku Pharmaceutical University, Miyagi, Japan. ${ }^{7}$ Present address: Ibaraki Prefectural Hospital, Ibaraki, Japan.

Received: 14 July 2015 Accepted: 23 December 2015

Published online: 05 January 2016

References

1. Janssens JP, Krause KH. Pneumonia in the very old. Lancet Infect Dis. 2004:4:112-24.

2. Gant V, Parton S. Community-acquired pneumonia. Curr Opin Pulm Med. 2000;6:226-33.

3. Segal AW. How neutrophils kill microbes. Annu Rev Immunol. 2005;23:197-223.

4. Briles DE, Claflin JL, Schroer K, Forman C. Mouse lgg3 antibodies are highly protective against infection with Streptococcus pneumoniae. Nature. 1981;294:88-90.

5. Miyasaka T, Akahori Y, Toyama M, Miyamura N, Ishii K, Saijo S, et al. Dectin2-dependent NKT cell activation and serotype-specific antibody production in mice immunized with pneumococcal polysaccharide vaccine. PLoS One. 2013;8:e78611. doi:10.1371/journal.pone.0078611.

6. Dallaire F, Ouellet N, Bergeron Y, Turmel V, Gauthier MC, Simard M, et al Microbiological and inflammatory factors associated with the development of pneumococcal pneumonia. J Infect Dis. 2001;184:292-300.

7. Saijo S, Ikeda S, Yamabe K, Kakuta S, Ishigame H, Akitsu A, et al. Dectin-2 recognition of alpha-mannans and induction of Th17 cell differentiation is essential for host defense against Candida albicans. Immunity. 2010;32:681-91.

8. Sato K, Yang XL, Yudate T, Chung JS, Wu J, Luby-Phelps K, et al. Dectin-2 is a pattern recognition receptor for fungi that couples with the Fc receptor gamma chain to induce innate immune responses. J Biol Chem. 2006;281:38854-66. 
9. Aggarwal S, Ghilardi N, Xie MH, de Sauvage FJ, Gurney AL. Interleukin-23 promotes a distinct CD4 T cell activation state characterized by the production of interleukin-17. J Biol Chem. 2003;278:1910-4.

10. Langrish CL, Chen Y, Blumenschein WM, Mattson J, Basham B, Sedgwick JD, et al. IL-23 drives a pathogenic T cell population that induces autoimmune inflammation. J Exp Med. 2005;201:233-40.

11. Harrington LE, Hatton RD, Mangan PR, Turner H, Murphy TL, Murphy KM, et al. Interleukin 17-producing CD4+ effector T cells develop via a lineage distinct from the Thelper type 1 and 2 lineages. Nat Immunol. 2005;6:1123-32.

12. Mills $\mathrm{KH}$. Induction, function and regulation of IL-17-producing T cells. Eur J Immunol. 2008;38:2636-49.

13. Yamamoto N, Kawakami K, Kinjo Y, Miyagi K, Kinjo T, Uezu K, et al. Essential role for the p40 subunit of interleukin-12 in neutrophil-mediated early host defense against pulmonary infection with Streptococcus pneumoniae: involvement of interferon-gamma. Microbes Infect. 2004;6:1241-9.

14. Sun K, Salmon SL, Lotz SA, Metzger DW. Interleukin-12 promotes gamma interferon-dependent neutrophil recruitment in the lung and improves protection against respiratory Streptococcus pneumoniae infection. Infect Immun. 2007;75:1196-202.

15. Rubins JB, Pomeroy C. Role of gamma interferon in the pathogenesis of bacteremic pneumococcal pneumonia. Infect Immun. 1997;65:2975-7.

16. Musher D, Cohen M, Baker C. Immune responses to extracellular bacteria. In: Rich RR, Fleisher TA, Schwartz BD, Shearer WT, Strober W, editors. Clinical immunology, Principles and Practice. St, Louis: Mosby-Year Book; 1996. p. 479-502.

17. Romero-Steiner S, Musher DM, Cetron MS, Pais LB, Groover JE, Fiore AE, et al. Reduction in functional antibody activity against Streptococcus pneumoniae in vaccinated elderly individuals highly correlates with decreased IgG antibody avidity. Clin Infect Dis. 1999;29:281-8.

18. Perlmutter RM, Hansburg D, Briles DE, Nicolotti RA, Davie JM. Subclass restriction of murine anti-carbohydrate antibodies. J Immunol. 1978;121:566-72.

19. Dessing MC, Schouten M, Draing C, Levi M, von Aulock S, van der Poll T. Role played by Toll-like receptors 2 and 4 in lipoteichoic acid-induced lung inflammation and coagulation. J Infect Dis. 2008;197:245-52.

20. Dessing MC, Hirst RA, de Vos AF, van der Poll T. Role of Toll-like receptors 2 and 4 in pulmonary inflammation and injury induced by pneumolysin in mice. PLoS One. 2009;4:e7993. doi:10.1371/journal.pone.0007993.

21. Echchannaoui H, Frei K, Schnell C, Leib SL, Zimmerli W, Landmann R. Tolllike receptor 2-deficient mice are highly susceptible to Streptococcus pneumoniae meningitis because of reduced bacterial clearing and enhanced inflammation. J Infect Dis. 2002;186:798-806.

22. Albiger B, Dahlberg S, Sandgren A, Wartha F, Beiter K, Katsuragi H, et al. Tolllike receptor 9 acts at an early stage in host defense against pneumococcal infection. Cell Microbiol. 2007;9:633-44.

23. Austrian R, Gold J. Pneumococcal bacteremia with especial reference to bacteremic pneumococcal pneumonia. Ann Intern Med. 1964;60:759-76

24. Usinger WR, Lucas AH. Avidity as a determinant of the protective efficacy of human antibodies to pneumococcal capsular polysaccharides. Infect Immun. 1999;67:2366-70.

25. Tian H, Weber S, Thorkildson P, Kozel TR, Pirofski LA. Efficacy of opsonic and nonopsonic serotype 3 pneumococcal capsular polysaccharide-specific monoclonal antibodies against intranasal challenge with Streptococcus pneumoniae in mice. Infect Immun. 2009;77:1502-13.

26. Mond JJ, Lees A, Snapper CM. T cell-independent antigens type 2. Annu Rev Immunol. 1995;13:655-92.

27. Shriner AK, Liu H, Sun G, Guimond M, Alugupalli KR. IL-7-dependent B lymphocytes are essential for the anti-polysaccharide response and protective immunity to Streptococcus pneumoniae. J Immunol. 2010;185:525-31.

28. Kobrynski LJ, Sousa AO, Nahmias AJ, Lee FK. Cutting edge: antibody production to pneumococcal polysaccharides requires CD1 molecules and CD8+ T cells. J Immunol. 2005;174:1787-90.

29. Verbinnen B, Covens K, Moens L, Meyts I, Bossuyt X. Human CD20 + CD43 + CD27+ CD5- B cells generate antibodies to capsular polysaccharides of Streptococcus pneumoniae. J Allergy Clin Immunol. 2012;130:272-5.

30. Haas KM, Poe JC, Steeber DA, Tedder TF. B-1a and B-1b cells exhibit distinct developmental requirements and have unique functional roles in innate and adaptive immunity to S. pneumoniae. Immunity. 2005;23:7-18.

31. Snapper CM, Mclntyre TM, Mandler R, Pecanha LM, Finkelman FD, Lees A, et al. Induction of IgG3 secretion by interferon gamma: a model for T cellindependent class switching in response to T cell-independent type 2 antigens. J Exp Med. 1992;175:1367-71.
32. Marchi LF, Sesti-Costa R, Ignacchiti MD, Chedraoui-Silva S, Mantovani B. In vitro activation of mouse neutrophils by recombinant human interferongamma: increased phagocytosis and release of reactive oxygen species and pro-inflammatory cytokines. Int Immunopharmacol. 2014;18:228-35.

33. Lee RT, Hsu TL, Huang SK, Hsieh SL, Wong CH, Lee YC. Survey of immunerelated, mannose/fucose-binding C-type lectin receptors reveals widely divergent sugar-binding specificities. Glycobiology. 2011;21:512-20.

34. Kennedy JF, Rosevear A. An assessment of the fractionation of carbohydrates on concanavalin A-sepharose 4B by affinity chromatography. J Chem Soc Perkin 1. 1973;19:2041-6.

35. Zamze S, Martinez-Pomares L, Jones H, Taylor PR, Stillion RJ, Gordon S, et al. Recognition of bacterial capsular polysaccharides and lipopolysaccharides by the macrophage mannose receptor. J Biol Chem. 2002;277:41613-23.

36. McGreal EP, Rosas M, Brown GD, Zamze S, Wong SY, Gordon S, et al. The carbohydrate-recognition domain of Dectin-2 is a C-type lectin with specificity for high mannose. Glycobiology. 2006;16:422-30.

37. Kawakami K, Yamamoto N, Kinjo Y, Miyagi K, Nakasone C, Uezu K, et al. Critical role of Va14 ${ }^{+}$natural killer $T$ cells in the innate phase of host protection against Streptococcus pneumoniae infection. Eur J Immunol. 2003;33:3322-30.

38. Kawakami K, Kohno S, Morikawa N, Kadota J, Saito A, Hara K. Activation of macrophages and expansion of specific T lymphocytes in the lungs of mice intratracheally inoculated with Cryptococcus neoformans. Clin Exp Immunol. 1994;96:230-7.

\section{Submit your next manuscript to BioMed Central and we will help you at every step:}

- We accept pre-submission inquiries

- Our selector tool helps you to find the most relevant journal

- We provide round the clock customer support

- Convenient online submission

- Thorough peer review

- Inclusion in PubMed and all major indexing services

- Maximum visibility for your research

Submit your manuscript at www.biomedcentral.com/submit
BioMed Central 